http://jmscr.igmpublication.org/home/ ISSN (e)-2347-176x ISSN (p) 2455-0450

crossref DOI: https://dx.doi.org/10.18535/jmscr/v8i3.100

Journal Of Medical Science And Clinical Research

\title{
Staphylococcal Scalded Skin Syndrome in an 11-Month-Old Child: A Case Report
}

\author{
Authors \\ Dr Aaron George ${ }^{1}$, Dr Greeshma B Susan ${ }^{2}$, Dr Jinumol Joy ${ }^{3}$, \\ Dr Elizebeth Civia Vincent ${ }^{4}$, Dr Linsa Linet $\mathbf{V}^{5}$ \\ ${ }^{1}$ Department of Pediatrics, St.James Hospital, Chalakudy, Paediatician \\ ${ }^{2,3,4,5}$ Pharm D Intern, St.James Hospital Chalakudy
}

\begin{abstract}
Staphylococcal scalded skin syndrome is caused by staphylococcus aureus. This can be seen in the children below 6 years, but can also affect adults with a depressed immune system or problem with kidney disease. This patient presented to us with fever and peeling of skin, and investigations showed lymphocytosis and skin culture was positive to staphylococcus aureus. The child was treated with ampicillin and improved.
\end{abstract}

\section{Introduction}

Staphylococcal scalded skin syndrome (SSSS) is an illness characterised by red blistering skin that looks like a burn or scald, hence called as staphylococcal scalded skin syndrome. SSSS has also been called Ritter disease or Lyell disease when it appears in newborns or young infants ${ }^{(1)}$. We came across with such a case in an eleven month old boy in the pediatric department.

\section{Case Presentation}

Here we present a case of 11 month old boy with blistering skin, erythrema.He had no relevant past medical and medication history prior to this event. No allergies were known.

\section{1-3 days}

He came to ED with loss of appetite, urticaria, periorbitaledema, and blisters at groin area since 2 days. Hehad complaints of pain in buttocks, lower back and face. Initially erythrema, perioral crusting and peeling of skin was not present. Child was apyretic. Due to periorbital edema and absence of mucous membrane involvement, perioraloedema and erythrema we came to working diagnosis as angioedema or pyoderma. Exfoliation affected approximately $10 \%$ of the TBSA. Laboratory test were done including blood routine, CRP. The blood results reveals the signs of infections; TC-19500/cumm, CRP:1.7 MG/DL. N39 L69 further investigations were done including skin culture.

We started antibiotic therapy with intravenous ceftriaxone, amikacin and urticaria was managed with emollients, anti-histamines and topical antibiotics. On third day he had desquamation of superficial layer of the skin when rubbed (nicolsky's sign).Since the child developed perioral crusting without mucous membrane involvement we suspected ssss and waited for the culture report. During these days feeding was reduced hence we added DNS 500 ML q6h. 


\section{4-6 days}

Culture results showed staphylococcus aureus .From the above signs along with the culture report we came to a final diagnosis as ssss. We changed antibiotic therapy intravenous ceftriaxone to inj. Ampicillin along with inj.amikacin and external application with emollients. Child had antibiotic induced diarrhea and managed with probiotics and $\mathrm{Zn}$.

Most of the blisters, urticaria and erythrema started to resolve and continued IV therapy along with local application of emolients. Affected TBSA decreased and child was symptomatically better. We repeated the serology, blood routine and found to be normal also patient's vitals were stable.

On time of discharge, pain over the buttocks had subsided and majority of the skin peeling had healed without scarring. Blisters were completely cured. On 6 th day child was found to be healthy, clinically stable and only slight skin peeling was present .He got discharged with oral antibiotics, probiotic and $\mathrm{Zn}$.
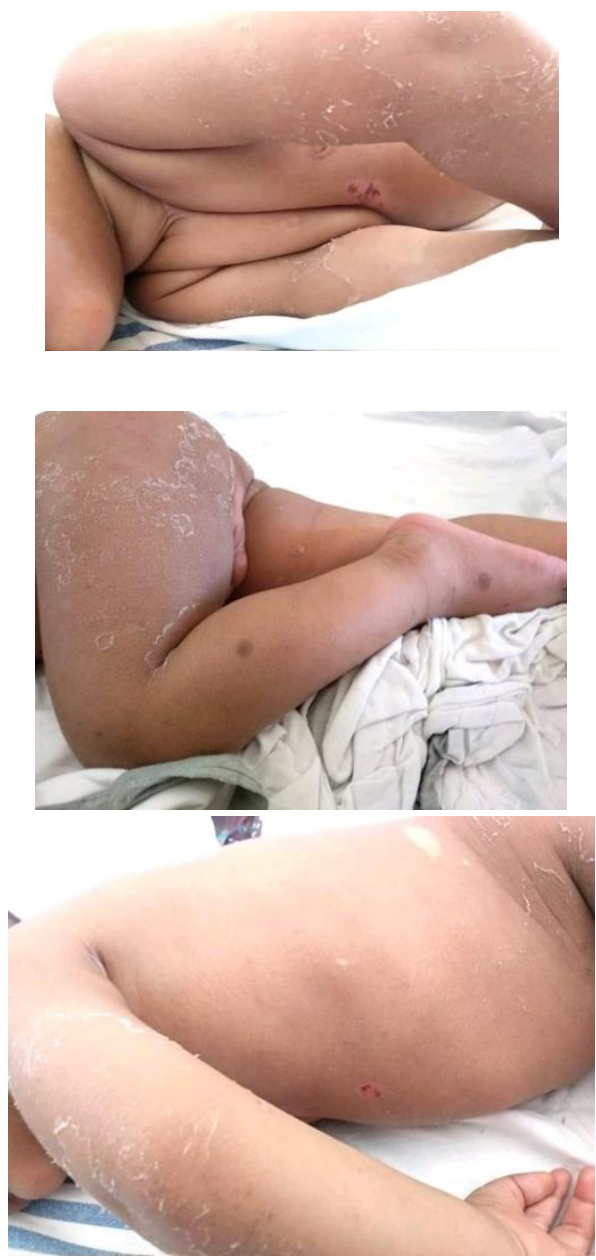

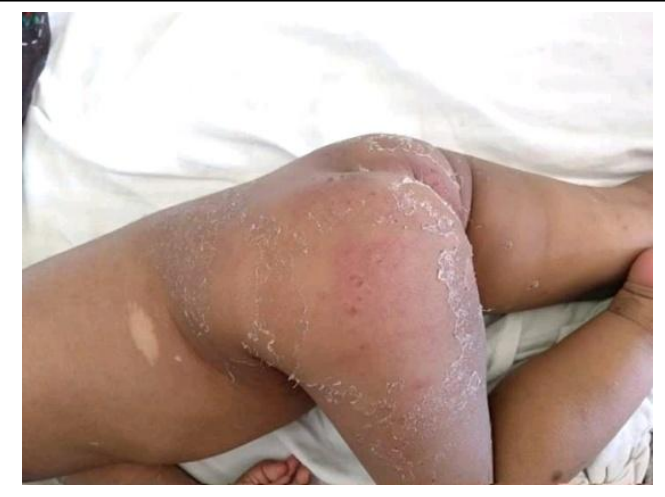

\section{Discussion}

In this case we had to clear the diagnosis of SSSS. The intravenous antibiotic therapy was effective in the patient and slight skin peeling was the only symptom persisting at the time of discharge.

SSSS is the rare skin condition which primarily affects children having remarkable blistering on superficial suface of skin. It was first described by the germanphysician, von Rittershain in 1878. Also called Ritter's disease is a rare affecting up to 56 people out of 100000 . It is most common in children under the age $6^{(2)}$. The statistical calculations indicated a low overall incidence between 0.09 and 0.13 cases per 1 million inhabitants per year, i.e., one case per 10 million inhabitants per year. Staphylococccus bacteria produces 2 types of toxins which include epidermolytic toxins $\mathrm{A}$ and $\mathrm{B}^{(4)}$. These toxins leads to the cleavage of desmoglein complex 1.Disintegration of desmosomes anchoring the strata granulose cause exfoliation ${ }^{(5)}$.

Complications of SSSS includes Pneumonia, dehydration, sepsis, shock like burn person, scar and death if untreated. Dehydration can lead to electrolyte imbalance therefore fluid management and laboratory values monitoring is important. Also Use of skin creams or ointments are recommended. ${ }^{(5,6)}$

Diagnostic method include History and physical examination, Skin biopsy, which shows intraepidermal cleavage at the granular layer, Bacterial culture from skin, blood, urine or umblical cord sample (in a newborn baby) ${ }^{(7)}$.

Treatment of SSSS usually requires hospitalisation, as intravenous antibiotics are generally necessary to eradicate the 
staphylococcal infection. A penicillinase-resistant, anti-staphylococcal antibiotic such as flucloxacillin is used. Other antibiotics include nafcillin, oxacillin, cephalosporin and clindamycin. Vancomycin is used in infections suspected with methicillin resistance (MRSA) ${ }^{(7)}$. Symptoms of sss can be similar to other desquamous disease like toxic epidermal necrolysis, SJS. Symptoms of SSSS can resemble those for other skin disorders such as bullous impetigo and certain forms of eczem ${ }^{(1)}$.

Differentiation is mainly done by assessing mucosal involvement. Lack of dermal inflammation indicates SSSS.TEN and SJS causes full thick exfoliation. That the cleavage site in staphylococcal scalded skin syndrome is intraepidermal, as opposed TEN, which involves necrosis of the full epidermal layer (at the level of the basement membrane) $)^{(3,5)}$

\section{Conclusion}

This case report emphasize on the diagnostic and therapeutic method of SSSS. We have discussed diagnostic methods and therapeutic options. From the above data we conclude that SSSS is a rare skin syndrome which is more prevalent in infants and children up to the age of 6 . The untreated condition can lead to complication and even death. The main treatment option include penicillinase resistant, anti- staphylococcal antibiotic. Clindamycin is considered as drug of choice to stop the production of exotoxin from bacteria.

\section{References}

1. Shamezladhani, staphylococcal scalded skin syndrome, BMJ, volume 78, issue 1.

2. Ross A, Staphylococcal scalded skin syndrome, statpearls [internet]; 2020 Jan.

3. Jeremy A, SJS and TEN; a concise review with a comprehensive summary of therapeutic interventions emphasizing supportive measures, advances in therapy, 2017;34(6):1235-1244.

4. P J Haasnoot, staphylococcal scalded skin syndrome in a 4 year old child ; a case report, journal of medical case report, Jan 2018,

5. Rare disease database.

6. Arun $\mathrm{k}$ Mishra,A systemic review on staphylococcal scalded skin syndrome (SSSS);A rare and critical disease for neonates, the open microbiology journal, august 2016,volume 10.pg no;150-159.

7. Medscape database. 